Chronic Obstructive Pulmonary Diseases: Journal of the COPD Foundation

\author{
Original Research
}

\title{
Psychometric Properties of the COPD-Specific Beliefs About Medicine Questionnaire in an Outpatient Population: A Rasch- Analysis
}

\author{
Marie Topp, $\mathrm{PhD}^{1}$ Jørgen Vestbo, DMSc ${ }^{2}$ Erik Lykke Mortensen, $\mathrm{MS}^{3}$
}

\begin{abstract}
Background: Understanding non-adherence in patients with chronic obstructive pulmonary disease (COPD) remains challenging. The necessity and the concerns scales of the Beliefs about Medicines Questionnaire (BMQ) are known to correlate with adherence behavior in several chronic diseases including asthma but less is known about COPD. In the present study a COPD-specific BMQ (BMQ-COPD) was translated and administered to Danish COPD outpatients. Our aim was to derive high-quality measures of the 2 dimensions included in the BMQ-COPD. Methods: A total of 168 patients with COPD were included from a Danish respiratory outpatient clinic. The Rasch model was used to evaluate psychometric characteristics of the BMQ-COPD and to obtain necessity and concerns scales fulfilling criteria of unidimensionality and overall fit, and with all items showing individual item fit with no local dependencies, and no differential item functioning. Further, we explored the association with disease-related variables and subsequent adherence behavior.

Results: Shortened versions of the original necessity and concerns scales fitted the Rasch model and were at least as good as the original scales as predictors of adherence, although only the necessity scale was significantly associated with adherence.

Conclusion: In a Danish COPD setting high-quality measures of necessity and concerns could be derived from the BMQ-COPD. The excluded items appear to reflect other dimensions.
\end{abstract}

\begin{abstract}
Abbreviations: chronic obstructive pulmonary disease, COPD; Beliefs about Medicines Questionaire, BMQ; COPD-specific Beliefs about Medicines Questionaire, COPD-BMQ; World Health Organiza-tion, WHO; Global initiative for chronic Obstructive Lung Disease, GOLD; long-acting mus-carinic, LAMA; inhaled corticosteroid, ICS; long-acting beta2-agonists, LABA; COPD Assessment Test, CAT; proportion of days covered, PDC; partial credit model, PCM; standard deviation, SD; Person-Separation Index, PSI; differential item functioning, DIF; forced expiratory volume in 1 second, $\mathbf{F E V}_{\mathbf{1}}$; odds ratio, OR; modified Medical Research Council, mMRC

Funding Support: This research was funded by the Danish Lunge Association, the Danish Foundation TrygFonden and the Danish Health Ministry.

Date of Acceptance: June 15, 2016

Citation: Topp M, Vestbo J, Mortensen EL. Psychometric properties of the COPD-specific Beliefs about Medicine Questionnaire in an outpatient population: A Rasch-analysis. Chronic Obstr Pulm Dis (Miami). 2016;3(4):748-757. doi: http://dx.doi.org/10.15326/ jcopdf.3.4.2016.0134
\end{abstract}

1 Department of Respiratory Medicine, Hvidovre Hospital, Denmark

2 Respiratory and Allergy Research Group, Manchester Academic Health Science Centre, University Hospital South Manchester, NHS Foundation Trust, Manchester, United Kingdom
3 Department of Public Health and Center for Healthy Aging, University of Copenhagen, Denmark 


\section{Address correspondence to:}

Marie Topp, $\mathrm{PhD}$

Kettegaard Alle 30

2650 Hvidovre, Denmark

E-mail: marie_topp@hotmail.com

Phone: +4561463089

\section{Keywords:}

chronic obstructive pulomonary disease; COPD; health beliefs; concerns; adherence; scale validation; unidimensionality

\section{Introduction}

Good adherence to inhaled therapy in patients with chronic diseases leads to improved disease control and reduced morbidity, hospitalization, and health care expenditures. ${ }^{1}$ According to the World Health Organization (WHO), patient adherence to longterm treatment in chronic diseases averages 50\% in developed countries. The level of adherence to prescribed inhaled medicine in patients with chronic obstructive pulmonary disease (COPD) is consistently low, often $<50 \%-60 \%$. ${ }^{2,3}$

The key to design interventions to improve medication adherence is a greater understanding of the factors related to poor adherence. ${ }^{4}$ The WHO emphasizes the patient perspective as 1 of 5 important aspects influencing adherence behavior, in addition to social and economic factors, health care systems, characteristics of the disease, and disease therapies. ${ }^{5}$

The existing knowledge about adherence in patients with COPD is not sufficient for a comprehensive description of patient heterogeneity and the corresponding diversity in adherence behavior. ${ }^{6}$ Several studies have attempted to demonstrate correlations between adherence and patient and disease characteristics ${ }^{7,8}$ but results have been inconsistent and do not indicate that patient and disease characteristics explain adherence behavior. In contrast, studies of other chronic diseases have consistently found a relationship between adherence and the individual's perceptions of need for medicine and concerns about potential adverse effects of medication. ${ }^{3,9,10}$ However, in COPD patients there is a need for studies of individual differences with regard to beliefs about medication and the influence of such beliefs on adherence behavior.

Horne and colleagues ${ }^{11}$ developed a multiitem questionnaire to measure necessities and concerns about prescribed medicine - the Specific Beliefs about Medicines Questionnaire (BMQ-specific). It is a selfreport, multi-item scale with 2 subscales assessing patients' perceptions of their need for medicine and concerns regarding medicine, respectively. The BMQ scores have been shown to correlate with adherence behavior in several chronic diseases including asthma but less is known about COPD. ${ }^{11,12}$ Consequently, Horne and colleagues further developed a COPDspecific BMQ (BMQ-COPD), which is not yet published. When introducing a multi-item scale it is vital to validate the scale in the specific population in which it will be used before the scale scores are used in clinical or research contexts. ${ }^{13}$ The Rasch model is increasingly being used to evaluate psychometric characteristics of multiitem scales and examines very important criteria for assessing the psychometric quality of a scale. ${ }^{13,14}$

The overall aim of this study was to derive high-quality measures of the necessity and the concerns scales of the COPD-specific BMQ in a Danish secondary health care outpatient population, and thus examine whether the 2 scales or parts of the scales fit a Rasch model. In addition, we analyzed the relationship between the 2 scores and the relationship between the scores and clinical assessments. Furthermore, we explored the potential difference between the original and the validated scales in the association with adherence behavior.

\section{Materials and Methods Participants and Design}

Participants were enrolled in an observational adherence study in the outpatient clinic of the Section of Respiratory Medicine at Hvidovre Hospital, Denmark. Out of 1919 patients followed in the clinic, we consecutively asked 335 patients to participate, if they had been prescribed one or more COPD maintenance medication for regular use. They were all previously diagnosed with COPD according to the Global initiative for chronic Obstructive Lung Disease (GOLD) ${ }^{15}$ and 168 patients were recruited (February 2011 to May 2013). Exclusion criteria were non-ability to read and understand questionnaires and tests applied during the adherence study. The study was approved by Hvidovre Hospital, the Danish Data Protection Agency and the regional scientific ethics committees for the Copenhagen area (H-3-2010-123). All participants gave written informed consent before participation. Information about demographics, smoking history, and prescribed respiratory medication and further medication was registered at inclusion. BMQ-COPD was one among several self-report questionnaires and tests included in the adherence study. Participants completed 
the questionnaire on their own with guidance available if needed. This scale-validation concentrates only on BMQ-COPD. On the basis of the COPD medication prescribed, the BMQ-COPD was completed in regard to 1 specific inhaler for maintenance treatment. We restricted analyses to long-acting muscarinic agents (LAMA) (ATC: R03BB), combinations of inhaled corticosteroids (ICS) and long-acting beta-agonists (LABA) (ATC: R03AK), and long-acting beta-agonists (LABA) (ATC: R03AC). If prescribed, a LAMA was the preferred option for the questionnaire as tiotropium was the most frequently prescribed drug for these patients.

\section{Measures}

The BMQ-COPD is a 22-item self-report questionnaire that consists of 2 subscales:

1) The necessity scale explores the respondent's beliefs about the need for specific inhaled medicine and includes 7 items. Total sum score has a maximum range from 7 to 35, and item names are: N1-N7 (N7 is reversely scored).

2) The concerns scale explores the respondent's beliefs about worries about taking their medicine and includes 15 items. Total sum-score has a maximum range from 15 to 75 , and the item names are: C1-15 (C8, C10, and C11 are reversely scored). Both scales are answered on a 5-point Likert scale ranging from "strongly disagree" to "strongly agree." All items relate to prescribed inhaled therapy and higher sum scores indicate stronger beliefs about necessity and concerns. The BMQ-COPD is developed from the Specific Belief about Medicines Questionnaire (BMQ) previously validated. ${ }^{11}$ The BMQ-COPD was translated and used with prior authorization of the developer. The authors conducted the first translation and professional translators produced the following back-translation. The orig-inal version, the translation and the backtranslation were discussed with the professional translators and the translation of items was modified until they were considered comparable to the original version. A pilot-test of the final Danish version on other patients from the outpatient clinic did not suggest further changes.

The COPD Assessment Test (CAT) aims to assess the impact of COPD on patients' daily-life and consists of 8 items scored from 0 (no impact) to 5 (very severe impact). High score indicates more severe impact of COPD $(<10$ : Low; 10-20: Medium; 21-30: High; >30: Very high). CAT provides a valid, reliable and standardized measure of COPD health status. ${ }^{16-20}$ The responsiveness of CAT is good, and it is used increasingly in clinical practice as well as clinical research in preference to more timeconsuming and complex questionnaires such as the St. George's Respiratory Questionnaire (SGRQ) and the Chronic Respiratory disease Questionnaire (CRQ). ${ }^{16-22}$

Refill-adherence to maintenance COPD medication in a 1-year observation period from study entry was estimated using data from the national Danish Register of Medicinal Product Statistics (of the Danish Medicines Agency), ${ }^{23}$ which holds information on every prescription dispensed in Danish pharmacies. We calculated the Proportion of Days Covered (PDC) for each prescribed medication matching the inhalers to the questionnaires for which questions related to the inhalers were answered. Overlapping refills and medication claimed up to 3 months before study entry were taken into account.

\section{Statistical Analysis}

By conducting a Rasch analysis we tested whether the sum of the item scores in the 2 multi-item scales necessity and concerns were actually measuring necessity and concerns, respectively, and thus valid in our specific population. The Rasch model is the simplest item response model having only 1 parameter per person and 1 parameter corresponding to each category of an item.

Rasch models have equal discrimination parameters, but models for items in Likert format differ with respect to assumptions about the thresholds for moving from one answer category to the next. The partial credit model (PCM) fits separate thresholds for each item and was used for both scales. For each item, the order of the thresholds has to be consistent with the answer categories. This is tested with a likelihood ratio test.

The item-trait interaction was used to evaluate the fit between model and data, reflecting the hierarchical order of items across the trait. Statistically nonsignificant probability value ( $p>0.05$; chi-square) indicates model fit. Additionally, fit-residual statistics were used for items and persons, reflecting the degree of divergence of the residuals between the expected and observed data for each person-item. A perfect model fit is indicated by residuals with a mean of 0.00 and a standard deviation (SD) of 1.00 .

Individual item misfit was determined to the model by item fit-residual values (residuals \pm 2.50 ) and item chisquare values ( $p<0.05$, Bonferroni adjusted). Item misfit 
suggests that the item reflects more than 1 dimension and these items were excluded.

The Person-Separation Index (PSI) indicates the power of the scale to discriminate amongst the respondents. The higher the PSI the better the discrimination power (PSI $>0.70$ is acceptable).

To evaluate the unidimensionality of each BMQ-scale we generated 2 subsets of items with positive versus negative correlations with the first principal component derived from the matrix of residual correlations. We tested the 2 subsets with a paired t-test for each person. To support the assumption of unidimensionality the number of significant tests should be less than $5 \%$ of the total number of tests.

Differential item functioning (DIF) occurs when subgroups respond differently to an item despite similar underlying levels of health beliefs. We performed the analyses of DIF by variance-based statistics for 2 demographic variables (gender and age) and for 2 disease-related variables (GOLD grade and CAT score). ${ }^{15,16}$

To evaluate local independency among items we used the correlations matrix of residuals for all items. A residual correlation between 0.2 and 0.3 above the average of all item residual correlations was defined as local dependency, which leads to evaluation of item redundancies and potentially, exclusion of 1 of the items.

We evaluated the internal consistency reliability with Cronbach's Alpha, ranging from 0-1 with 0.70 being the lowest level of acceptability.

Items were excluded one by one by deleting the most insignificant item followed by repeated item fit statistics for the remaining items until every item in the scale fitted the model. The individual item selections were simultaneously evaluated in relation to content validity, unidimensionality, local independencies, and DIF.

In addition to the Rasch analysis, we examined the Pearson correlation between the vali-dated necessity and concern scores. To further explore the relationship between the scores, the 2 scales were split at the scale midpoints to generate 4 attitudinal groups: 1 ) sceptical (low necessity, high concerns); 2) indifferent (low necessity, low concerns; 3) ambivalent (high necessity, high concerns); 4) accepting (high necessity, low concerns). ${ }^{24}$ In addition, we examined the Pearson correlation between the necessity and the concern scores in relation to forced expiratory volume in 1 second $\left(\mathrm{FEV}_{1}\right)$ in \% and CAT score.
Furthermore, to compare the validated scales and the original scales we examined the association between the scale scores and refill-adherence in a logistic regression analysis with dichotomized refill-adherence as outcome, adjusted for age, lung function and socioeconomic status. Due to very high adherence rates in the study population we chose to analyze the effect of beliefs about medicines on good adherence compared to perfect adherence by using a 95\% cut-point for the dichotomization.

The Rasch analysis was conducted with the program RUMM2030. Statistical analyses were performed using statistical SAS 9.3.

\section{Results \\ Participants}

A total of 167 of the 168 participants completed the BMQ-COPD. Half of the participants had severe COPD, and none of the participants had mild COPD. Almost $80 \%$ of the patients were former smokers and only 3 patients had never smoked. The mean age was 68.6 years. Detailed characteristics are presented in Table 1. The majority of the patients were prescribed LAMAs and or ICS/LABA combinations, and the BMQ-COPD questionnaire was answered in regard to LAMAs by 135 patients, for ICS/LABAs by 26 patients, and for LABAs by 6 patients, respectively.

\section{Necessity Scale}

The 7-item necessity scale did not fit the overall RSM $(p<0.0001)$. Individual item fit failed for item N5, "This inhaler protects me from becoming worse," and item N7, "I cannot feel this inhaler working." The remaining 5 items fit the PCM $(p=0.213$, PSI=0.85) and comprised the final necessity scale, cf. (Table 2) The 5-item scale showed unidimensionality, with no local dependencies, and the orders of all item thresholds were consistent. None of the 5 items showed DIF in regard to age, gender, CAT score and GOLD grade. Cronbach's alpha was 0.89 .

\section{Concerns Scale}

The 15-item concerns scale did not fit the PCM $(p<0.00001)$. Individual item-fit to the model failed for 5 out of 15 items (item C8, C10-C12, C15, see Table 3), but the remaining 10 items did not show unidimensionality ( $9 \%$ of the tests showed $p<0.05$ ). After combining tests for unidimensionality, tests of individual item fit and evaluation of content validity, we 
decided to exclude item C13 and C14 and achieved an overall fit, individual item fit, local independencies and unidimensionality with the remaining 8 items. Thus, item C1-C7 and item C9 comprised the final scale (overall test of fit for the PCM: $p=0.81$, PSI $=0.85$, test of unidimensionality: $4.19 \%$ of the tests showed $p<0.05$ ), Table 3.

The 8 items in the final scale did not show DIF with regard to age, gender, CAT score and GOLD grade. Cronbach's alpha was 0.83 . The threshold order was not consistent for item 3, 4, 6 and 7 in categories 4 and

\section{Table 1. Demographic and Patient Characteristics}

\begin{tabular}{|c|c|}
\hline Gender n (\%) & \\
\hline Male & $70(41.7)$ \\
\hline Female & $98(58.3)$ \\
\hline Mean Age (min-max) & $68.6(42.3-85.2)$ \\
\hline \multicolumn{2}{|l|}{ Smoking Status n (\%) } \\
\hline Never Smoked & $3(1.8)$ \\
\hline Former Smoker & $132(78.6)$ \\
\hline Current Smoker & $31(18.5)$ \\
\hline Special Occasion Smoking & $2(1.2)$ \\
\hline \multicolumn{2}{|l|}{ mMRC n (\%) } \\
\hline Score 1 & $9(5.4)$ \\
\hline Score 2 & $50(29.8)$ \\
\hline Score 3 & $64(38.1)$ \\
\hline Score 4 & $41(24.4)$ \\
\hline Score 5 & $4(2.4)$ \\
\hline \multicolumn{2}{|l|}{ GOLD n (\%) } \\
\hline Moderate COPD (FEV ${ }_{1}$ 50-80) & $43(25.6)$ \\
\hline Severe COPD (FEV $130-50)$ & $81(48.2)$ \\
\hline Very Severe $\left(\mathrm{FEV}_{1}<30\right)$ & $44(26.2)$ \\
\hline Mean FEV $_{1} \%$ Predicted (min-max) & $40.2(11.6-75.0)$ \\
\hline Mean CAT Score (min-max) & $17.0(2.0-38.0)$ \\
\hline \multicolumn{2}{|l|}{ Comorbidity } \\
\hline $0-1$ & $59(37.8)$ \\
\hline$>2$ & $97(62.2)$ \\
\hline
\end{tabular}

5 (agree and strongly agree). However, there were few responses in these categories (2 to 12 responses).

\section{Relation Between Necessity and Concerns}

The mean value was 18.7 for the 5 -item necessity scale and 20.5 for the 8 -item concerns scale. Only $12 \%$ of the patients reported low level of necessity. Almost one third of the patients reported ambivalent attitude, while $60 \%$ of the patients reported accepting attitude (Figure 1 ). The correlation between the scales was close to zero and the correlations between each scale and clinical measurements showed positive but weak relationship between the necessity score and CAT score ( $r=0.17$, $p=0.027)$. In addition, the CAT score correlated with $\mathrm{FEV}_{1}$ predicted $(\mathrm{r}=0.23, p=0.0024)$.

\section{Comparing the Original and Validated Versions of the Necessity and Concerns Scale}

As expected, the correlations between the original and the Rasch-validated scales were very strong for the necessity scale $(\mathrm{r}=0.96, p<0.0001)$ as well as for the concerns scale $(\mathrm{r}=0.90, p<0.0001)$. The shorter validated necessity scale was as least as good at predicting adherence to inhaled maintenance medication, (odds ratio $[O R]=0.90$ per scale point $p=0.04)$ as the original necessity scale $(\mathrm{OR}=0.92, p=0.04)$. Neither the shorter validated concerns scale ( $\mathrm{OR}=1.0, p=0.99)$ nor the original concerns scale ( $\mathrm{OR}=1.01, p=0.74)$ predicted adherence over 1 year in our patient sample.

\section{Discussion}

Using Rasch analysis we obtained a shortened version of the COPD-specific Beliefs about Medicines Questionnaire with 2 well-functioning scales measuring patients' beliefs of necessity for prescribed inhaled medicine and patients' concerns about their medicine. Due to multi-unidimensionality and item misfit in our

\section{Table 2. Necessity Scale Items and Individual Item Fit}

\begin{tabular}{|c|c|c|c|c|}
\hline $\begin{array}{l}\text { Item } \\
\text { Name }\end{array}$ & Item Question & $\begin{array}{l}\text { Test- } \\
\text { value }\end{array}$ & p-value & $\begin{array}{c}\text { Order of } \\
\text { Exclu-sion }\end{array}$ \\
\hline N1 & My health, at present, depends on this inhaler & 0.65 & 0.11 & Final scale \\
\hline N2 & My life would be impossible without this inhaler & -2.44 & 0.57 & Final scale \\
\hline N3 & Without this inhaler I would be very ill & -0.87 & 0.54 & Final scale \\
\hline N4 & My health in the future will depend on this inhaler & -0.67 & 0.80 & Final scale \\
\hline N5 & This inhaler protects me from becoming worse & 2.79 & 0.002 & 2 \\
\hline N6 & This inhaler is the most important part of my treatment & 1.98 & 0.05 & Final scale \\
\hline N7 & I CANNOT feel this inhaler working & 7.13 & $<0.000001$ & 1 \\
\hline
\end{tabular}




\section{Table 3. Concerns Scale Items and Individual Item Fit}

\begin{tabular}{|c|c|c|c|c|}
\hline $\begin{array}{l}\text { Item } \\
\text { Name }\end{array}$ & Item Question & Residual & p-value & $\begin{array}{l}\text { Order of } \\
\text { Exclusion }\end{array}$ \\
\hline C1 & Having to use this inhaler worries me & 0.54 & 0.90 & Final scale \\
\hline $\mathbf{C 2}$ & $\begin{array}{l}\text { I sometimes worry about the long-term effects of this } \\
\text { inhaler }\end{array}$ & 0.82 & 0.87 & Final scale \\
\hline $\mathbf{C 3}$ & This inhaler is a mystery to me & 1.60 & 0.40 & Final scale \\
\hline $\mathbf{C 4}$ & This inhaler disrupts my life & 0.28 & 0.80 & Final scale \\
\hline C5 & $\begin{array}{l}\text { I sometimes worry about becoming too dependent on } \\
\text { this inhaler }\end{array}$ & -1.76 & 0.18 & Final scale \\
\hline C6 & This inhaler does more harm than good & -0.12 & 0.27 & Final scale \\
\hline C7 & $\begin{array}{l}\text { People who are on this inhaler should stop their } \\
\text { treatment every now and again. }\end{array}$ & -0.44 & 0.69 & Final scale \\
\hline $\mathbf{C 8 r}$ & $\begin{array}{l}\text { I have been given enough information about my } \\
\text { treatment }\end{array}$ & 3.44 & $<0.00001$ & 1 \\
\hline C9 & $\begin{array}{l}\text { I am concerned that this inhaler might become less } \\
\text { effective if I use it regularly }\end{array}$ & 1.45 & 0.49 & Final scale \\
\hline C10r & This inhaler is harmless & 3.64 & 0.0002 & 3 \\
\hline C11r & $\begin{array}{l}\text { I can use as much of this inhaler as I need without } \\
\text { having to worry about becoming too dependent on it }\end{array}$ & 4.87 & $<0.00001$ & 2 \\
\hline C12 & This inhaler gives me unpleasant side-effects & 3.07 & 0.079 & 5 \\
\hline C13 & Using this inhaler is embarrassing & 1.77 & $0.023^{\mathrm{a}}$ & 6 \\
\hline C14 & This inhaler is difficult to use & 0.41 & $0.014^{\mathrm{b}}$ & 7 \\
\hline C15 & $\begin{array}{l}\text { I sometimes do not have enough breath to use this } \\
\text { inhaler }\end{array}$ & 3.42 & 0.0006 & 4 \\
\hline
\end{tabular}

a excluded despite residual $<2.5$ and $p>0.005$ (Bonferroni adjusted $p<0.05$ for 10 items)

b excluded despite residual $<2.5$ and $p>0.0056$ (Bonferroni adjusted $p<0.05$ for 9 items)

Rasch model, several items were excluded from the original scales. The validated necessity scale correlated well with the CAT score and the validated scales were found to be at least as good as the original scales to predict adherence behavior.

Treating the growing number of COPD patients is challenging because of non-adherence to medications as well as other aspects of disease management. In our current treatment paradigm long-acting bronchodilators, inhaled corticosteroids and, to some extent, phosphodiesterase-4 inhibitors are all used to prevent exacerbations ${ }^{25,26}$ and require good adherence. ${ }^{1}$ Simple tools are needed in a clinical setting to enable health professionals to address non-adherence and the BMQ-COPD offers the possibility to explore the needs and concerns of patients. Health beliefs are increasingly discussed in research on adherence behavior and patients' beliefs about medicine seems to relate to adherence behavior in asthma but less is known about COPD. ${ }^{12,27,28}$ We found it very relevant to explore the possible causal relationship between beliefs about prescribed inhaled medicine and level of adherence since perceived drug effects and longterm expectations may differ significantly between asthma and COPD. Different questionnaires have been used to evaluate beliefs about medicine. ${ }^{29-31}$ As the BMQ-COPD is the only COPD-specific "beliefs about medicine" inventory, it was an obvious choice. However, before using the sum scores from the multi-item scales, a systematic scale validation is essential.

In our analysis the necessity scale was reduced by 2 items (Item N5 and N7). We would have expected item N5 to fit the model since the content of the item ("My inhaler protects me from becoming worse") seems comparable to item N4 ("My health in the future depends on this inhaler"); nevertheless, the itemmisfit was substantial for N5. It probably reflects that patients are able to distinguish between the inescapable progression of COPD irrespective of inhaled medication and the fact that they know that the inhaled medication 


\section{Figure 1.Relation Between the Necessity and the Concerns Scale}

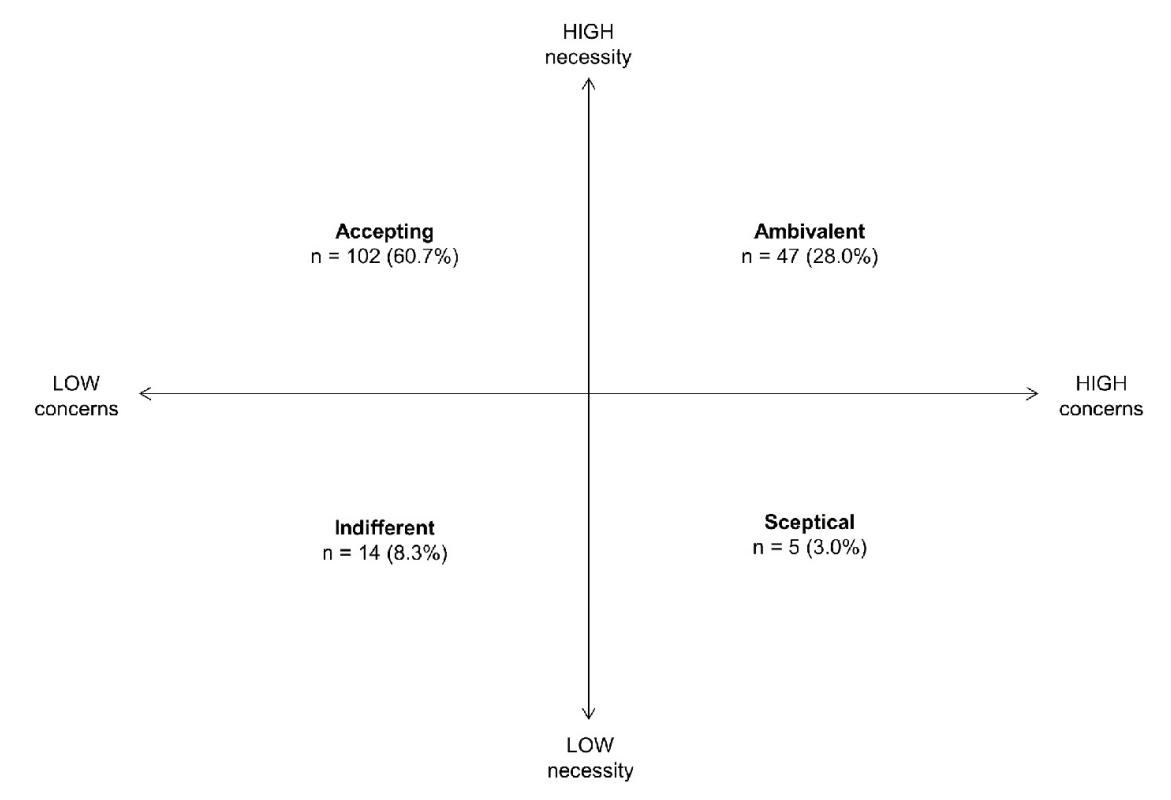

Item C10 ("This inhaler is harmless") and C11 ("I can use as much of this inhaler as I need, without having to worry about becoming too dependent on it") both seem content valid to the concern issue, but their formulation requires reverse scoring and this may confuse the respondents. It may reflect translation problems, but may also reflect misunderstanding of reversed questions. ${ }^{32}$ The excluded 9 items may still correlate with adherence levels as well as with the necessity and concerns scales; however, they appear to reflect more dimensions than the dimensions assessed by the 2 scales.

On the final scales only $12 \%$ of the participants reported a low degree of necessity. More than half of the participants belonged in the accepting attitude category and we

is important for their future health. The fact that Item N7 ("I cannot feel this inhaler") did not fit the model indicates that the beliefs about the necessity of the medicine seem different from the patient's perception of relief in symptoms. Furthermore, most patients perceive more immediate substantial symptom relief with short-term inhalers than the maintenance inhalers that were the subject of the BMQ-COPD questionnaire. The necessity scale in the non-disease-specific BMQ contains only 5 items 11 ; item N6 and N7 were added in the BMQ-COPD version. Thus, item N5 ("These medicines protect me from becoming worse") has been shown to fit the multi-item scale in the BMQ, but the BMQ-COPD item N5 ("This inhaler protects me from be-coming worse") does not fit our model. This suggests that COPD patients in general are aware of the likely worsening of their condition and the limitations of medical treatment.

The concerns scale was at first reduced by 5 items (items C8, C10-C12, C15) and by further analysis by 2 more items (items $\mathrm{C} 13$ and $\mathrm{C} 14$ ) resulting in an 8-item scale. Item $C 6, C 7$, and $C 9$ are additions to the non-disease-specific BMQ concerns scale but seem to be very relevant to describe the COPD specific concerns about inhaled therapy. Although item C12 seems relevant to COPD concerns, other items focus on information rather than medicine (item C8) or focus on actual inhaler use and technique (items C13-C15). would expect these participants to adhere better to their inhaler. In contrast, we expect that patients belonging in the ambivalent attitude category (28\%) could be in a dilemma weighing necessity and concerns. Absence of correlation between the 2 scores confirms that the 2 scales measure separate dimensions. The positive correlation between the necessity score and the CAT score is clinically meaningful and interesting, and tells us that there is a relationship between perception of necessity and experienced symptom burden. However, the correlation is relatively weak and this suggests that perception of necessity is probably determined by other factors than the clinical state of the patients. This may explain why classical clinical evaluation does not predict adherence very well. ${ }^{3}$

The shorter validated scales were at least as good as the original scales at predicting adherence, although only the necessity scales were associated with adherence in this study population. However, the difference in the association with adherence behavior in this large sample is not convincing, but if the scales are used to evaluate individual patients in a clinical context, the Rasch validation could have significant impact on the necessity and the concerns measure. Furthermore, for use in clinical practice the shorter form is a very important advantage of the validated scales.

Our study has limitations. The respondents to the BMQ-COPD volunteered to participate in an adherence 
study, which makes possible selection bias an issue. The participants are thought to be comparable to the patients with COPD in our outpatient clinic in general as indicated by similar variation in GOLD grade, MRC, etc. ${ }^{33}$ However, although representative of secondary care outpatients, our study population has more severe disease and may be more homogeneous than that seen in primary care. This may have implications if this tool is to be used in primary care where it would seem necessary to check the validity of the scales as well. Also, the findings may only be representative of patients with a similar exposure to inhaled drugs as they are prescribed in our clinic, a general COPD clinic in Northern European secondary care. Furthermore, responders completing the questionnaire regarding different inhaled medications could bias our findings, as the choice of bronchodilators versus corticosteroid could influence the outcome of BMQ-COPD. This is, however, somewhat contradicted by the fact that it was actually one of the perceived relief questions (item N5) that did not fit the necessity scale. Finally, the high adherence level in the study population limited our possibility to evaluate differences in the association with adherence between the original and the validated scales.

We conclude that the COPD-specific Beliefs about Medicine Questionnaire represents 2 multi-item scales assessing patients' perception of their need for medicine and patients' concerns regarding their medicine, respectively. In a Danish COPD outpatient setting the validat-ed scales were reduced with 7 items in total. The excluded items appear to reflect other dimensions than those reflected in the necessity and concerns scales. The sum-scores of the validated scales did not show strong correlation with measures of disease burden indicating that the perception of necessity and concerns about prescribed inhaled medicines is influenced by other factors. The shorter validated scales were at least as good in predicting adherence behavior as the original scales and the questionnaire is likely to become an important tool for future research on the relationship between beliefs about medicine and adherence behavior in patients with COPD.

\section{Aknowledgements}

The authors would like to thank Professor Robert Horne for providing us access to the COPD specific Beliefs about Medicines Questionnaire.

\section{Declaration of Interest}

Dr. Vestbo reports personal fees from GlaxoSmithKline, personal fees from Chiesi Pharmaceuticals, personal fees from Boehringer-Ingelheim, personal fees from Novartis, personal fees from Almirall, personal fees from AstraZeneca, outside the submitted work. Drs. Topp and Mortensen have nothing to declare. 


\section{References}

1. Vestbo J, Anderson JA, Calverley PM, et al. Adherence to inhaled therapy, mortality and hospital admission in COPD. Thorax. 2009;64(11):939-943.

doi: http://dx.doi.org/10.1136/thx.2009.113662

2. Krigsman K, Moen J, Nilsson JL, Ring L. Refill adherence by the elderly for asthma/chronic obstructive pulmonary disease drugs dispensed over a 10-year period. J Clin Pharm Ther. 2007;32(6):603-611.

doi: http://dx.doi.org/10.1111/j.1365-2710.2007.00866.x

3. Rand CS. Patient adherence with COPD therapy. Eur Respir Rev. 2005;14(96):97-101.

doi: http://dx.doi.org/10.1183/09059180.05.00009604

4. Ownby RL, Hertzog C, Czaja SJ. Relations between cognitive status and medication adherence in patients treated for memory disorders. Ageing Res. 2012;3(1):e2.

doi: http://dx.doi.org/10.4081/ar.2012.e2

5. Sabaté E, World Health Organization. Adherence to Long-term Therapies - Evidence for Action. Geneva, Switzerland: World Health Organization; 2003. http://www.who.int/chp/knowledge/ publications/adherence_full_report.pdf?ua=1

Accessed July 2016.

6. Haynes RB, Ackloo E, Sahota N, McDonald HP, Yao X. Interventions for enhancing medication adherence. Cochrane Database Syst Rev. 2008(2):CD000011.

7. Agh T, Inotai A, Meszaros A. Factors associated with medication adherence in patients with chronic obstructive pulmonary disease. Respiration. 2011;82(4):328-334.

doi: http://dx.doi.org/10.1159/000324453

8. Bourbeau J, Bartlett SJ. Patient adherence in COPD. Thorax. 2008;63(9):831-838.

doi: http://dx.doi.org/10.1136/thx.2007.086041

9. Horne R, Weinman J. Patients' beliefs about prescribed medicines and their role in adherence to treatment in chronic physical illness. J Psychosom Res. 1999;47(6):555-567. doi: http://dx.doi.org/10.1016/s0022-3999(99)00057-4

10. DiMatteo MR, Haskard KB, Williams SL. Health beliefs, disease severity, and patient adherence: a meta-analysis. Med Care. 2007;45(6):521-528.

doi: http://dx.doi.org/10.1097/MLR.0b013e318032937e

11. Horne R, Weinman J, Hankins M. The beliefs about medicines questionnaire: The development and evaluation of a new method for assessing the cognitive representation of medication. Psychol Health. 1999;14(1):1-24.

doi: http://dx.doi.org/10.1080/08870449908407311

12. Krauskopf K, Federman AD, Kale MS, et al. Chronic obstructive pulmonary disease illness and medication beliefs are associated with medication adherence. COPD. 2015;12(2):151-164. doi: http://dx.doi.org/10.3109/15412555.2014.922067
13. Fayers PM, Manchin D. Quality of Life. Assessment, Analysis and Interpretation. Chichester, England: John Wiley \& Sons Ltd.; 2007.

14. Hays RD, Morales LS, Reise SP. Item response theory and health outcomes measurement in the 21st century. Med Care. 2000; 38(9 Suppl).

doi: http://dx.doi.org/10.1097/00005650-200009002-00007

15. Global Initiative for Chronic Obstructive Lung Disease (GOLD). Global Strategy for the Diagnosis, Management and Prevention of COPD, 2015. GOLD website http://www.goldcopd.org/ uploads/users/files/GOLD_Report_2015.pdf Accessed February 2015. Published 2015.

16. Jones PW, Harding G, Berry P, Wiklund I, Chen WH, Kline Leidy N. Development and first validation of the COPD Assessment Test. Eur Respir J. 2009;34(3):648-654. doi: http://dx.doi.org/10.1183/09031936.00102509

17. Jones PW, Brusselle G, Dal Negro RW, et al. Properties of the COPD assessment test in a cross-sectional European study. Eur Respir J. 2011;38(1):29-35.

doi: http://dx.doi.org/10.1183/09031936.00177210

18. Kon SS, Canavan JL, Jones SE, et al. Minimum clinically important difference for the COPD Assessment Test: a prospective analysis. Lancet Respir Med. 2014;2(3):195-203. doi: http://dx.doi.org/10.1016/S2213-2600(14)70001-3

19. Jones PW, Nadeau G, Small M, Adamek L. COPD assessment test --rationale, development, validation and performance. Characteristics of a COPD population categorised using the GOLD framework by health status and exacerbations. COPD. 2013;10:269-271.

doi: http://dx.doi.org/10.3109/15412555.2013.776920

20. Gupta N, Pinto LM, Morogan A, Bourbeau J. The COPD assessment test: a systematic review. Eur Respir J. 2014;44(4):873884. doi: http://dx.doi.org/10.1183/09031936.00025214

21. Ringbaek T, Martinez G, Lange P. A comparison of the assessment of quality of life with CAT, CCQ, and SGRQ in COPD patients participating in pulmonary rehabilitation. COPD. 2012;9(1):1215. doi: http://dx.doi.org/10.3109/15412555.2011.630248

22. Jones PW, Harding G, Wiklund I, et al. Tests of the responsiveness of the COPD assessment test following acute exacerbation and pulmonary rehabilitation. Chest. 2012;142(1):134-140. doi: http://dx.doi.org/10.1378/chest.11-0309

23. Danish Medicines Agency. Register of Medicinal Product Statistics. Danish Medicines Agency website. https:// laegemiddelstyrelsen.dk/en/

Accessed July 2016. Published 2016.

24. Horne R, Parham R, Driscoll R, Robinson A. Patients' attitudes to medicines and adherence to maintenance treatment in inflammatory bowel disease. Inflamm Bowel Dis. 2009;15(6):837844. doi: http://dx.doi.org/10.1002/ibd.20846 
25. Vestbo J, Hurd SS, Agusti AG, et al. Global strategy for the diagnosis, management, and prevention of chronic obstructive pulmonary disease: GOLD executive summary. Am J Respir Crit Care Med. 2013;187(4):347-365.

doi: http://dx.doi.org/10.1164/rccm.201204-0596PP

26. National Institute for Health and Care Excellence (NICE). Chronic obstructive pulmonary disease: managment of chronic obstructive pulmonary disease in adults in primary and secondary care. NICE website. https://www.nice.org.uk/ guidance/cg12 Published February 2004. Accessed September 14, 2016.

27. Horne R, Weinman J. Self-regulation and self-management in asthma: exploring the role of illness perceptions and treatment beliefs in explaining non-adherence to preventer medication. Psychol Health. 2002;17(1):17-32. doi: http://dx.doi.org/10.1080/08870440290001502

28. Horne R. Compliance, adherence, and concordance: implications for asthma treatment. Chest. 2006;130(1 Suppl):65S-72S. doi: http://dx.doi.org/10.1378/chest.130.1_suppl.65S

29. Khdour MR, Hawwa AF, Kidney JC, Smyth BM, McElnay JC. Potential risk factors for medication non-adherence in patients with chronic obstructive pulmonary disease (COPD). Eur J Clin Pharmacol. 2012;68(10):1365-1373. doi: http://dx.doi.org/10.1007/s00228-012-1279-5

30. Rosser BA, Lm M, Velleman SC, Boichat C, Eccleston C. Concerns about medication and medication adherence in patients with chronic pain recruited from general practice. Pain. 2011;152(5):1201-1205.

doi: http://dx.doi.org/10.1016/j.pain.2011.01.053

31. Apter AJ, Reisine ST, Affleck G, Barrows E, ZuWallack RL. Adherence with twice-daily dosing of inhaled steroids. Socioeconomic and health-belief differences. Am J Respir Crit Care Med. 1998;157(6 Pt 1):1810-1817.

doi: http://dx.doi.org/10.1164/ajrccm.157.6.9712007

32. Barnette JJ. Effects of stem and likert response option reversals on survey internal concistency: If you feel the need, there is a better alternative using those negatively worded stems. (Statistical Data Included). Educ Psychol Meas. 2000;60(3):361. doi: http://dx.doi.org/10.1177/00131640021970592

33. DrKOL. Danish COPD register. Copenhagen: National Board of Health; 2012. 